YITP/U- 91-43

October, 1991

\title{
3-dimensional Gravity from the Turaev-Viro Invariant
}

\author{
Shun'ya Mizoguchi[] \\ and \\ TSUKASA TADA \\ Uji Research Center \\ Yukawa Institute for Theoretical Physics \\ Kyoto University, Uji 611 Japan
}

\begin{abstract}
We study the $q$-deformed su(2) spin network as a 3-dimensional quantum gravity model. We show that in the semiclassical continuum limit the Turaev-Viro invariant obtained recently defines naturally regularized path-integral à la Ponzano-Regge, In which a contribution from the cosmological term is effectively included. The regularization dependent cosmological constant is found to be $\frac{4 \pi^{2}}{k^{2}}+O\left(k^{-4}\right)$, where $q^{2 k}=1$. We also discuss the relation to the Euclidean Chern-Simons-Witten gravity in 3-dimension.
\end{abstract}

\footnotetext{
${ }^{\dagger}$ JSPS fellow. e-mail: mizo@jpnrifp.yukawa.kyoto-u.ac.jp

${ }^{\ddagger}$ Soryuushi shogakukai fellow. e-mail: tada@yisun1.yukawa.kyoto-u.ac.jp
} 
It is well-known that 3-dimensional gravity is perturbatively trivial in the sense that there are no local degrees of freedom. In 3-dimension Ricci-flat space-time means truly flat space-time, so that there are no gravitational wave modes but is only topological excitation. Topological nature of 3-dimensional gravity has already been encountered in the old work by Ponzano and Regge on the semi-classical limit of the Racah coefficients of $\mathrm{su}(2)[\mathbb{1}$. In their seminal paper (and also ref. [2]) a triangulation-independent quantity was defined by utilizing a certain relation for $6 j$ symbols, and was shown to be viewed as a path-integral for 3-dimensional quantum gravity in the semi-classical continuum limit, though their expression diverges and needs some regularization. Recently, Turaev and Viro has constructed a new topological invariant from the $q$-deformed $\mathrm{su}(2)$ spin network when $q$ is a root of unity. Their construction strongly resembles that of ref. [1], and moreover, the Turaev-Viro (TV) invariant is naturally regularized and finite due to the restriction for the spin variables. Therefore the TV invariant is expected to be considered as a regularized path-integral for 3-dimensional quantum gravity.

In this letter we estimate the asymptotic behavior of the $q-6 j$ symbol by the WKB approximation along the method of ref. [3], and see how the path-integral defined by the $\mathrm{su}(2)$ spin network receives 'quantum' corrections from $q$-deformation. We show that a contribution from the cosmological term is effectively included in the path-integral from the TV invariant.

In ref. [1] a sum of products of four (classical) $\mathrm{su}(2)$ Racah-Wigner $6 j$ symbols was considered:

$$
\begin{aligned}
& \sum_{\substack{x, l_{1}, l_{2}, l_{3}: \\
\text { allowed value }}}(2 x+1)\left(2 l_{1}+1\right)\left(2 l_{2}+1\right)\left(2 l_{3}+1\right)(-1)^{\chi} \\
& \quad \times\left\{\begin{array}{ccc}
j_{1} & j_{2} & j_{3} \\
x & l_{1} & l_{2}
\end{array}\right\}\left\{\begin{array}{lll}
j_{6} & j_{5} & j_{1} \\
l_{1} & l_{2} & l_{3}
\end{array}\right\}\left\{\begin{array}{lll}
j_{4} & j_{2} & j_{6} \\
l_{2} & l_{3} & x
\end{array}\right\}\left\{\begin{array}{lll}
j_{3} & j_{5} & j_{4} \\
l_{3} & x & l_{1}
\end{array}\right\},
\end{aligned}
$$

where $\chi=x+\sum_{i=1}^{3} l_{i}+\sum_{i=1}^{6} j_{i}$. By repeated application of the Biedenharn-Elliott identity and the orthogonal relation, (1) is reduced to a single $6 j$ symbol:

$$
\sum_{x=0}^{\infty}(2 x+1)^{2}\left\{\begin{array}{lll}
j_{1} & j_{2} & j_{3} \\
j_{4} & j_{5} & j_{6}
\end{array}\right\} .
$$

To give a meaning to the divergent expression (2) a large angular-momentum cut-off $R$ is introduced in the summation, so that we take the equality between (11) and (2) as the 
following renormalized identity:

$$
\begin{aligned}
& \left\{\begin{array}{lll}
j_{1} & j_{2} & j_{3} \\
j_{4} & j_{5} & j_{6}
\end{array}\right\} \\
= & \lim _{R \rightarrow \infty} \frac{3 a}{4 R^{3}} \sum_{x, l_{1}, l_{2}, l_{3}<R}(2 x+1)\left(2 l_{1}+1\right)\left(2 l_{2}+1\right)\left(2 l_{3}+1\right)(-1)^{\chi} \\
& \times\left\{\begin{array}{lll}
j_{1} & j_{2} & j_{3} \\
x & l_{1} & l_{2}
\end{array}\right\}\left\{\begin{array}{lll}
j_{6} & j_{5} & j_{1} \\
l_{1} & l_{2} & l_{3}
\end{array}\right\}\left\{\begin{array}{lll}
j_{4} & j_{2} & j_{6} \\
l_{2} & l_{3} & x
\end{array}\right\}\left\{\begin{array}{lll}
j_{3} & j_{5} & j_{4} \\
l_{3} & x & l_{1}
\end{array}\right\},
\end{aligned}
$$

where $a$ is some dimensionless constant (finite renormalization). Since a $6 j$ symbol is naturally associated to a tetrahedron, the identity (3) means that the value of $6 j$ associated to a tetrahedron is invariant under subdivision if the summation is carried out over all allowed lengths of internal edges with appropriate weight (Fig.1). Repeating the subdivision (3), we find

$$
\begin{aligned}
& \left\{\begin{array}{lll}
j_{1} & j_{2} & j_{3} \\
j_{4} & j_{5} & j_{6}
\end{array}\right\} \\
= & \lim _{R \rightarrow \infty}\left(\frac{3 a}{4 R^{3}}\right)^{e} \sum_{x_{1}<R} \cdots \sum_{x_{f}<R} \prod_{i=1}^{d}\left[T_{i}\right] \\
& \times(-1)^{\sum_{l=1}^{6} j_{l}} \prod_{p=1}^{f}(-1)^{x_{p}}\left(2 x_{p}+1\right) \\
\equiv & I_{P R},
\end{aligned}
$$

where

$$
\begin{aligned}
{\left[T_{i}\right] \quad: } & 6 j \text { symbol assigned to the } i \text { th simplex } \\
d, e, f: & \text { the number of simplices,internal vertices } \\
& \text { and internal edges,respectively } \\
x_{p}: & \text { (the length of the } p \text { th edge })-\frac{1}{2} .
\end{aligned}
$$

Hence $I_{P R}$ may be taken as a 'topological invariant', where topological invariant means that it does not depend on the triangulation inside the 3-manifold, but depends only on the edge-lengths on the boundary and topology of the triangulated 3-manifold. Obviously, with fixed boundaries, $I_{P R}$ takes the same value in each class of triangulation connected through the operation (3). 
In the semi-classical continuum limit $x_{p}, e$ (and so $f$ ) go to infinity. The $6 j$ symbol behaves as [1, [3]

$$
\left\{\begin{array}{lll}
j_{1} & j_{2} & j_{3} \\
j_{4} & j_{5} & j_{6}
\end{array}\right\} \approx\left(\frac{1}{12 \pi V}\right)^{\frac{1}{2}} \cos \left(\sum_{i=1}^{f} \theta_{i} J_{i}+\frac{\pi}{4}\right)
$$

in the domain where $J_{i}$ are uniformly large (see Figure Caption). Here $V$ is the volume of the tetrahedron. Thus one may write $I_{P R}$ as

$$
I_{P R} \sim \int d \mu \prod_{\text {tetrahedra }} \cos \left(\sum_{i=1}^{f} x_{i}\left(\pi-\theta_{i}\right)-\frac{\pi}{4}\right)
$$

with

$$
d \mu=\left(\frac{3 a}{4 R^{3}}\right)^{e} \prod_{i=1}^{f} d x_{i}\left(2 x_{i}+1\right) .
$$

Taking only the positive frequency part of cosine, together with $\sum_{i=1}^{f} x_{i}\left(\pi-\theta_{i}\right) \sim \frac{1}{2} \int \sqrt{g} R$ 田, one finds

$$
I_{P R} \text { (positive frequency part) } \sim \int d \mu \exp \left(i \frac{1}{2} \int \sqrt{g} R\right) .
$$

The above consideration is an interesting possibility to relate $I_{P R}$ with $3 \mathrm{~d}$ quantum gravity, though there are some difficulties, i.e. the appearance of $i$ in the exponent and the treatment of the other interference terms.

Turaev and Viro defined the quantity $I_{T V}$ (up to the factors from the boundary) as [5]

$$
I_{T V} \equiv w^{-2 e} \sum_{\text {allowed } \phi} \prod_{i=1}^{d}\left|T_{i}^{\phi}\right| \prod_{i=1}^{f} w_{i}^{2}
$$

where $\left|T_{i}^{\phi}\right|, w_{i}$ and $w$ are weight assigned to tetrahedra, edges and vertices, respectively. $\phi$ stands for a configuration of edge-lengths, called coloring. $\phi$ is an allowed ('admissible' in ref. [5]) coloring if any set of triple which forms a face of a tetrahedron satisfies the triangle inequality, and if the sum of any triple is an integer less than $k-2$. They proved that $I_{T V}$ is independent of triangulation if

$$
\begin{aligned}
& \left|T_{i}^{\phi}\right|=(-1)^{\sum_{l=1}^{6} j_{l}^{(i)}}\left\{\begin{array}{ccc}
j_{1}^{(i)} & j_{2}^{(i)} & j_{3}^{(i)} \\
j_{4}^{(i)} & j_{5}^{(i)} & j_{6}^{(i)}
\end{array}\right\}_{q} \\
& w_{i}^{2}=\left[2 x_{i}+1\right]_{q}, \quad w^{2}=-\frac{2 k}{\left(q-q^{-1}\right)^{2}},
\end{aligned}
$$


where $\{\ldots\}$ stands for a (restricted) $q-6 j$ symbol with $q=e^{\frac{\pi i}{k}} \llbracket 6$, and $[n]_{q}$ is a $q$-integer $[n]_{q}=\frac{q^{n}-q^{-n}}{q-q^{-1}}$. If $q$ is a root of unity in general, some Clebsch-Gordan coefficients of the representation of $U_{q}(\mathrm{sl}(2))$ diverge. Therefore one must, and can successfully, restrict the domain of the spin variables to a finite set $\left(\left\{0, \frac{1}{2}, 1, \ldots, k-\frac{1}{2}\right\}\right.$ in our case).

It is surprising that $I_{P R}$ and $I_{T V}$ are in the same form. Moreover, since

$$
\begin{aligned}
\left\{\begin{array}{ccc}
j_{1}^{(i)} & j_{2}^{(i)} & j_{3}^{(i)} \\
j_{4}^{(i)} & j_{5}^{(i)} & j_{6}^{(i)}
\end{array}\right\}_{q} & =\left\{\begin{array}{ccc}
j_{1}^{(i)} & j_{2}^{(i)} & j_{3}^{(i)} \\
j_{4}^{(i)} & j_{5}^{(i)} & j_{6}^{(i)}
\end{array}\right\}+O\left(k^{-2}\right) \\
{\left[2 x_{i}+1\right]_{q} } & =2 x_{i}+1+O\left(k^{-2}\right) \\
-\frac{2 k}{\left(q-q^{-1}\right)^{2}} & =\frac{k^{3}}{2 \pi^{2}}\left(1+O\left(k^{-2}\right)\right)
\end{aligned}
$$

$I_{T V}$ approaches $I_{P R}$ in the $k \rightarrow \infty(q \rightarrow 1)$ limit with $a=\frac{8 \pi^{2}}{3}$, where $k$ plays the same role as $R$. In other word, the TV invariant $I_{T V}$ provides a natural regularized path-integral for 3-dimensional quantum gravity.

Now let us study the asymptotic behavior of $q-6 j$ symbols. We start from the following relation, which is a special case of quantum analogue of the Biedenharn-Elliot identity for restricted $q-6 j$ symbols $[6]^{\S}$ :

$$
\begin{aligned}
& \left\{\begin{array}{ccc}
j_{1} & j_{2} & j_{3} \\
j_{4} & j_{5} & j_{6}
\end{array}\right\}_{q}\left\{\begin{array}{ccc}
j_{1} & j_{2} & j_{3} \\
\frac{1}{2} & j_{3}+\Delta_{b} & j_{2}+\Delta_{a}
\end{array}\right\}_{q} \\
& =\sum_{\zeta=j_{4} \pm \frac{1}{2}}(-1)^{j_{1}+2 j_{2}+2 j_{3}+j_{4}+j_{5}+j_{6}+\zeta+\frac{1}{2}+\Delta_{a}+\Delta_{b}}[2 \zeta+1]_{q} \\
& \times\left\{\begin{array}{ccc}
j_{1} & j_{2}+\Delta_{a} & j_{3}+\Delta_{b} \\
\zeta & j_{5} & j_{6}
\end{array}\right\}_{q}\left\{\begin{array}{ccc}
\frac{1}{2} & j_{2} & j_{2}+\Delta_{a} \\
j_{6} & \zeta & j_{4}
\end{array}\right\}_{q} \\
& \times\left\{\begin{array}{ccc}
\frac{1}{2} & j_{3}+\Delta_{b} & j_{3} \\
j_{5} & j_{4} & \zeta
\end{array}\right\}_{q}\left(\Delta_{a, b}= \pm \frac{1}{2}\right) .
\end{aligned}
$$

Combining the equations above, one obtains the following recursion relation:

$$
\left[2 j_{1}\right]_{q} g\left(j_{1}+1\right)\left\{\begin{array}{ccc}
j_{1}+1 & j_{2} & j_{3} \\
j_{4} & j_{5} & j_{6}
\end{array}\right\}_{q}
$$

\footnotetext{
$\S$ Nomura also discussed identities of $q-6 j$ and asymptotic behavior when a part of the arguments is large [10].
} 


$$
\begin{aligned}
& +2 h\left(j_{1}\right)\left\{\begin{array}{lll}
j_{1} & j_{2} & j_{3} \\
j_{4} & j_{5} & j_{6}
\end{array}\right\}_{q} \\
& +\left[2 j_{1}+2\right]_{q} g\left(j_{1}\right)\left\{\begin{array}{ccc}
j_{1}-1 & j_{2} & j_{3} \\
j_{4} & j_{5} & j_{6}
\end{array}\right\}_{q}=0 \\
& g(j)=\left\{\left[j_{2}+j_{3}+1+j\right]_{q}\left[j_{2}+j_{3}+1-j\right]_{q}\right. \\
& \times\left[j+j_{2}-j_{3}\right]_{q}\left[j-j_{2}+j_{3}\right]_{q} \\
& \times\left[j_{5}+j_{6}+1+j\right]_{q}\left[j_{5}+j_{6}+1-j\right]_{q} \\
& \left.\times\left[j+j_{5}-j_{6}\right]_{q}\left[j-j_{5}+j_{6}\right]_{q}\right\}^{\frac{1}{2}} \\
& h(j)=\left\{[2 j+1]_{q}[2 j+2]_{q}[2 j]_{q}\right. \\
& \times\left[j_{3}+j_{4}+j_{5}+2\right]_{q}\left[j_{3}+j_{5}-j_{4}+1\right]_{q} \\
& -[2 j]_{q}\left[j+j_{5}+j_{6}+2\right]_{q}\left[j+j_{5}-j_{6}+1\right]_{q} \\
& \times\left[j+j_{2}+j_{3}+2\right]_{q}\left[j+j_{3}-j_{2}+1\right]_{q} \\
& -[2 j+2]_{q}\left[j_{6}-j_{5}+j\right]_{q}\left[j_{5}+j_{6}-j+1\right]_{q} \\
& \left.\times\left[j_{2}+j_{3}-j+1\right]_{q}\left[j-j_{2}+j_{3}\right]_{q}\right\} / 2 .
\end{aligned}
$$

Since we are considering restricted $q-6 j$ symbols, the spin variables are truncated at $k$. Therefore, to take large angular momentum limit we have to take $k$ also large. Hence we substitute $j_{1}, j_{2}, \ldots$ for $\lambda j_{1}, \lambda j_{2}, \ldots$ and $k$ for $\lambda k$ at the same time, then we send $\lambda \rightarrow \infty$, keeping the ratio $j_{i} / k \ll 1$. Defining the $q$-analog of triangle-area:

$$
F_{q}(a, b, c)=\frac{1}{4} \sqrt{[a+b+c]_{q}[a-b+c]_{q}[a+b-c]_{q}[-a+b+c]_{q}},
$$

we can express $g(j)$ in a more compact form:

$$
g(j)=16 F_{q}\left(j, j_{2}+\frac{1}{2}, j_{3}+\frac{1}{2}\right) F_{q}\left(j, j_{5}+\frac{1}{2}, j_{6}+\frac{1}{2}\right) .
$$

Recalling the distinction between $J$ and $j$ (Fig.1), we obtain

$$
\begin{aligned}
& g(\lambda j)=16 \sqrt{F_{q}\left(\lambda J, \lambda J_{2}, \lambda J_{3}\right) F_{q}\left(\lambda J-1, \lambda J_{2}, \lambda J_{3}\right) F_{q}\left(\lambda J, \lambda J_{5}, \lambda J_{6}\right) F_{q}\left(\lambda J-1, \lambda J_{5}, \lambda J_{6}\right)} \\
& \times\left[1+O\left(\lambda^{-2}\right)\right] .
\end{aligned}
$$

Thus we rewrite the relation (13), keeping terms up to order $\lambda^{-1}$, as follows:

$$
\left\{\frac{F_{q}\left(\lambda J_{1}+1, \lambda J_{2}, \lambda J_{3}\right) F_{q}\left(\lambda J_{1}+1, \lambda J_{5}, \lambda J_{6}\right)}{\left[2\left(\lambda J_{1}+1\right)\right]_{q}}\right\}^{\frac{1}{2}}\left\{\begin{array}{ccc}
\lambda j_{1}+1 & \lambda j_{2} & \lambda j_{3} \\
\lambda j_{4} & \lambda j_{5} & \lambda j_{6}
\end{array}\right\}_{q}
$$




$$
\begin{gathered}
+\left\{\frac{F_{q}\left(\lambda J_{1}-1, \lambda J_{2}, \lambda J_{3}\right) F_{q}\left(\lambda J_{1}-1, \lambda J_{5}, \lambda J_{6}\right)}{\left[2\left(\lambda J_{1}-1\right)\right]_{q}}\right\}^{\frac{1}{2}}\left\{\begin{array}{ccc}
\lambda j_{1}-1 & \lambda j_{2} & \lambda j_{3} \\
\lambda j_{4} & \lambda j_{5} & \lambda j_{6}
\end{array}\right\}_{q} \\
+\frac{h\left(\lambda j_{1}\right)}{8\left\{\left[2\left(\lambda J_{1}+1\right)\right]_{q}\left[2\left(\lambda J_{1}-1\right)\right]_{q}\right\}^{1 / 2}} \frac{\left\{F_{q}\left(\lambda J_{1}, \lambda J_{2}, \lambda J_{3}\right) F_{q}\left(\lambda J_{1}, \lambda J_{5}, \lambda J_{6}\right)\right\}^{-1 / 2}}{\left\{\left[2 \lambda J_{1}\right]_{q}\right\}^{1 / 2}} \\
\quad \times\left\{\begin{array}{lll}
\lambda j_{1} & \lambda j_{2} & \lambda j_{3} \\
\lambda j_{4} & \lambda j_{5} & \lambda j_{6}
\end{array}\right\}_{q}=0 .
\end{gathered}
$$

Introducing

$$
\begin{aligned}
\varphi\left(j_{1}\right) & \equiv\left\{\frac{F_{q}\left(\lambda J_{1}, \lambda J_{2}, \lambda J_{3}\right) F_{q}\left(\lambda J_{1}, \lambda J_{5}, \lambda J_{6}\right)}{\left[2 \lambda J_{1}\right]_{q}}\right\}^{1 / 2}\left\{\begin{array}{lll}
\lambda j_{1} & \lambda j_{2} & \lambda j_{3} \\
\lambda j_{4} & \lambda j_{5} & \lambda j_{6}
\end{array}\right\}_{q} \\
c\left(J_{1}\right) & \equiv-\frac{h\left(\lambda j_{1}\right)}{16\left\{\left[2\left(\lambda J_{1}+1\right)\right]_{q}\left[2\left(\lambda J_{1}-1\right)\right]_{q}\right\}^{1 / 2} F_{q}\left(\lambda J_{1}, \lambda J_{2}, \lambda J_{3}\right) F_{q}\left(\lambda J_{1}, \lambda J_{5}, \lambda J_{6}\right)}
\end{aligned}
$$

we arrive at a difference equation for $j_{1}$ :

$$
\left\{\Delta^{2}+2-2 c\left(J_{1}\right)\right\} \varphi\left(j_{1}\right)=0 .
$$

Let us estimate the solution of the above equation (22) in the large $k$ limit. When $q \rightarrow 1, c\left(J_{1}\right)$ approaches its 'classical' value $\cos \theta_{1}$ [3], so one expands $c\left(J_{1}\right)$ as

$$
c\left(J_{1}\right)=\cos \theta_{1}+\varrho+O\left(k^{-4}\right),
$$

where the next-leading term $\varrho$ is of order $k^{-2}$. Since

$$
\begin{aligned}
\cos \left(\theta-\frac{\varrho}{\sin \theta}\right) & =\cos \theta \cos \left(\frac{\varrho}{\sin \theta}\right)+\sin \theta \sin \left(\frac{\varrho}{\sin \theta}\right) \\
& =\cos \theta\left(1-\frac{\varrho^{2}}{2 \sin ^{2} \theta}+\ldots\right)+\sin \theta\left(\frac{\varrho}{\sin \theta}-\frac{\varrho^{3}}{3 ! \sin ^{3} \theta}+\ldots\right) \\
& =\cos \theta+\varrho+O\left(k^{-4}\right),
\end{aligned}
$$

(22) becomes in the large $k$ limit as follows:

$$
\left\{\Delta^{2}+2-2 \cos \left(\theta_{1}-\frac{\varrho}{\sin \theta_{1}}\right)\right\} \varphi(x)=0 .
$$

Here we have changed the variable from $J_{1}$ to $x$. According to ref. [3], we solve the difference equation (25) by WKB approximation. The result is

$$
\varphi(x) \approx \frac{C}{\sqrt{\sin \left(\theta_{1}-\frac{\varrho}{\sin \theta_{1}}\right)}} \cos \left(\int\left(\theta_{1}-\frac{\varrho}{\sin \theta_{1}}\right) d x+\frac{\pi}{4}\right),
$$


where $C$ is a normalization. Thus we obtain the expression for the asymptotic formula of $q-6 j$ symbol:

$$
\left\{\begin{array}{lll}
j_{1} & j_{2} & j_{3} \\
j_{4} & j_{5} & j_{6}
\end{array}\right\}_{q} \sim \frac{C^{\prime}}{\sqrt{V}} \cos \left(\int \theta_{1}(x) d x-\int \frac{\varrho(x)}{\sin \theta_{1}} d x+\frac{\pi}{4}\right) .
$$

In the above expression $C^{\prime}$ is a quantity that approaches to $C=\frac{1}{\sqrt{12 \pi}}$ as $q \rightarrow 1$ and could be a function of $J_{i}$ at $k^{-2}$ order. The second term inside the cosine corresponds to a regularization counter term, while the spin dependence of $C^{\prime}$ may be regarded as a correction for the measure. The integral of $\theta_{1}$ in the cosine is proved to be $\sum \theta_{i} J_{i}$ [3].

Next we estimate $\varrho / \sin \theta$ in the large $k$ and angular momenta limit. Defining $\epsilon_{q}=\frac{\pi^{2}}{6 k^{2}}$, then

$$
[\lambda J]_{q}=\left(\lambda J-\epsilon_{q} \lambda J^{3}\right)\left[1+O\left(\lambda^{-2}\right)\right]
$$

so that

$$
\begin{aligned}
c\left(J_{1}\right)= & -\frac{h\left(\lambda j_{1}\right)}{16\left\{\left[2\left(\lambda J_{1}+1\right)\right]_{q}\left[2\left(\lambda J_{1}-1\right)\right]_{q}\right\}^{1 / 2} F_{q}\left(\lambda J_{1}, \lambda J_{2}, \lambda J_{3}\right) F_{q}\left(\lambda J_{1}, \lambda J_{5}, \lambda J_{6}\right)} \\
= & -\frac{\lambda^{5}\left(h_{c}+\epsilon_{q} h_{q}\right)\left\{1+\epsilon_{q}\left(8 J_{1}^{2}+2\left(J_{2}^{2}+J_{3}^{2}+J_{5}^{2}+J_{6}^{2}\right)\right)\right\}}{2 \lambda J_{1} \cdot 16 F_{c}\left(\lambda J_{1}, \lambda J_{2}, \lambda J_{3}\right) F_{c}\left(\lambda J_{1}, \lambda J_{5}, \lambda J_{6}\right)} \\
= & \cos \theta_{1}-\epsilon_{q} \frac{h_{q}+\left(8 J_{1}^{2}+2\left(J_{2}^{2}+J_{3}^{2}+J_{5}^{2}+J_{6}^{2}\right)\right) h_{c}}{2 J_{1} \cdot 16 F_{c}\left(J_{1}, J_{2}, J_{3}\right) F_{c}\left(J_{1}, J_{5}, J_{6}\right)}+O\left(\lambda^{-2}\right)
\end{aligned}
$$

where $h_{c}$ and $h_{q}$ are the following:

$$
\begin{aligned}
h_{c}=-2 J_{1}\left(J_{1}^{4}-J_{1}^{2} J_{2}^{2}-J_{1}^{2} J_{3}^{2}+2 J_{1}^{2} J_{4}^{2}-J_{1}^{2} J_{5}^{2}\right. & \left.-J_{2}^{2} J_{5}^{2}+J_{3}^{2} J_{5}^{2}-J_{1}^{2} J_{6}^{2}+J_{2}^{2} J_{6}^{2}-J_{3}^{2} J_{6}^{2}\right) \\
h_{q}=4 J_{1}( & 4 J_{1}^{6}-3 J_{1}^{4} J_{2}^{2}-J_{1}^{2} J_{2}^{4}-3 J_{1}^{4} J_{3}^{2}-J_{1}^{2} J_{3}^{4}+12 J_{1}^{4} J_{4}^{2}+2 J_{1}^{2} J_{4}^{4}-3 J_{1}^{4} J_{5}^{2} \\
& -8 J_{1}^{2} J_{2}^{2} J_{5}^{2}-J_{2}^{4} J_{5}^{2}+2 J_{1}^{2} J_{3}^{2} J_{5}^{2}+J_{3}^{4} J_{5}^{2}-J_{1}^{2} J_{5}^{4}-J_{2}^{2} J_{5}^{4}+J_{3}^{2} J_{5}^{4}-3 J_{1}^{4} J_{6}^{2} \\
+ & \left.2 J_{1}^{2} J_{2}^{2} J_{6}^{2}+J_{2}^{4} J_{6}^{2}-8 J_{1}^{2} J_{3}^{2} J_{6}^{2}-J_{3}^{4} J_{6}^{2}-J_{1}^{2} J_{6}^{4}+J_{2}^{2} J_{6}^{4}-J_{3}^{2} J_{6}^{4}\right)
\end{aligned}
$$

$F_{c}(a, b, c)$ is the 'classical' value of $F_{q}(a, b, c)$, that is, the area of a triangle whose edges are of length $a, b$ and $c$.

The numerator of the second term of (29) proved to be:

$$
8 J_{1}^{3} \cdot 16 F_{c}\left(J_{4}, J_{2}, J_{6}\right) F_{c}\left(J_{4}, J_{5}, J_{3}\right) \cos \theta_{4}
$$


Using the formula

$$
\frac{\partial V}{\partial J_{1}}=-\frac{J_{1} \cdot 16 F_{c}\left(J_{4}, J_{2}, J_{6}\right) F_{c}\left(J_{4}, J_{5}, J_{3}\right) \cos \theta_{4}}{144 V},
$$

we obtain

$$
\begin{aligned}
\varrho & =-\epsilon_{q} \times \frac{8 J_{1}^{3} \cdot 16 F_{c}\left(J_{4}, J_{2}, J_{6}\right) F_{c}\left(J_{4}, J_{5}, J_{3}\right) \cos \theta_{4}}{2 J_{1} \cdot 16 F_{c}\left(J_{1}, J_{2}, J_{3}\right) F_{c}\left(J_{1}, J_{5}, J_{6}\right)} \\
& =24 \epsilon_{q} \frac{\partial V}{\partial J_{1}} \sin \theta_{1} .
\end{aligned}
$$

Inserting (34) into (27), we conclude that

$$
I_{T V} \text { (positive frequency part) } \sim \int d \mu^{\prime} \exp \left(i \frac{1}{2} \int \sqrt{g}\left(R-\frac{8 \pi^{2}}{k^{2}}\right)\right),
$$

where $d \mu^{\prime}=d \mu+O\left(k^{-2}\right)$.

It is a plausible result that the cosmological term appears as a regularization counter term though our approximation for $q-6 j$ symbol is valid only in the region $j_{i} \ll k$ and all the $j_{i}$ are uniformly large. If the sum-region with respect to edge-lengths is unbounded, any configuration with high deficit angles would be allowed. In our case the summation is truncated at length $k$ so that the configuration is chopped off if it is spiky enough; consequently, the virtual processes with large volume are suppressed.

A crucial question is the following: "what does it mean if you gather only negative frequency factors to obtain a 'Lorentzian' action ( $i \times$ classical action), though you have been considering a Euclidean system?" In $2+1$-dimension the Einstein action coincides with the Chern-Simons (CS) action with gauge group $G$ under some appropriate fieldidentification, where $G$ is the isometry group of space-time, i.e. $G=\operatorname{ISO}(2,1), \operatorname{SO}(3,1)$ and $\mathrm{SO}(2,2)$ if space-time is Minkowski, de-Sitter and anti-de-Sitter, respectively [7]. However, in 3-dimension with Euclidean signature the Lagrangian for the $\mathrm{SO}$ (4) CS theory, which is supposed to be the Euclidean version for space-time with positive cosmological constant, is pure imaginary; hence the equivalence to the Einstein gravity is subtle [7]. On the other hand, we started from the TV invariant and interpreted it as the path-integral in the semi-classical continuum limit. Since amplitude for any process is topologically invariant, so should be the action. Therefore it would be better to consider the Regge action appeared in our model as the CS action, rather than the Einstein 
action. The strange $i$ factor may indicate the subtlety in the correspondence between the Euclidean gravity and a CS theory.

Indeed, there is an evidence that the TV invariant is related to a CS theory. If one evaluates $I_{T V}$ in a 3-manifold $F \times[0,1]$ where $F$ is a triangulated 2-surface, then $I_{T V}$ naturally induces a representation of the modular group of $F$ [5]. Comparing the representation with that of the Jones polynomial, Turaev and Viro conjectured that their invariant would be related to the 'square' of the Hilbert space of a topological field theory for the Jones polynomial, i.e. the SU(2) CS theory with Wilson lines [8]. Recently, it has been shown in ref. [9] that the TV invariants can be constructed from the $\mathrm{SO}(4)$ $(=\mathrm{SU}(2) \times \mathrm{SU}(2))$ CS theory with Wilson lines in the large $k$ limit.

We would like to thank M. Ninomiya for very fruitful discussions and continuous encouragement. We also thank M. Hayashi, A. Hosoya, S. Iso, S. Sawada, J. Soda and H. Suzuki for discussions. One of us (T. T.) also thanks A. Kirillov and H. Ooguri for discussions, and appreciate a kind hospitality of RIMS 91 Project. This work is supported in part by JSPS and Soryuushi shogakukai.

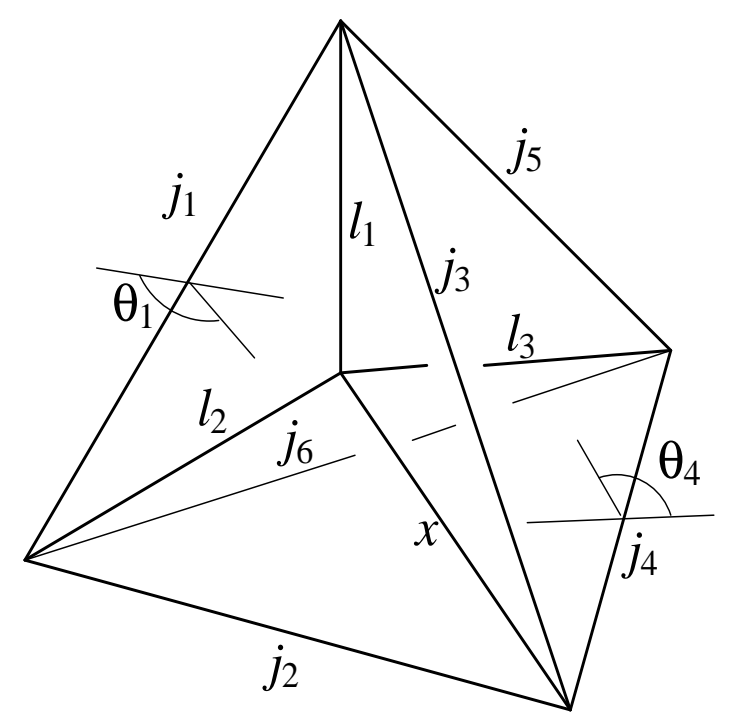

Fig.1. The $6 \mathrm{j}$ symbol $\left\{\begin{array}{ccc}j_{1} & j_{2} & j_{3} \\ j_{4} & j_{5} & j_{6}\end{array}\right\}$ is represented by a tetrahedron whose edges are of length $J_{i}=j_{i}+\frac{1}{2} . \theta_{i}$ is the angle between the outer normal of the two faces which have the edge $J_{i}$ in common. The subdivision (5) is also illustrated. 


\section{References}

[1] G. Ponzano and T. Regge, in:Spectroscopic and group theoretical methods in physics, ed. F. Bloch (North-Holland, Amsterdam, 1968).

[2] B. Hasslacher and M. J. Perry, Phys. Lett. B103 (1981) 21.

[3] K. Schulten and R. G. Gordon, Jour. Math. Phys. 16 (1975) 1961; ibid 1971.

[4] T. Regge, Nuovo Cimento 19 (1961) 551.

[5] V. G. Turaev and O. Y. Viro, LOMI preprint.

[6] A. N. Kirillov and N. Yu. Reshetikhin, in Adv. Ser. in Math. Phys, ed. G. Kac, vol. 7 (1988).

[7] E. Witten, Nucl. Phys. B311 (1988) 46.

[8] E. Witten, Commun. Math. Phys.121(1989)351.

[9] H. Ooguri and N. Sasakura, KUNS-1088, August 1991.

[10] M. Nomura, J. Math. Phys. 30 (1989) 2397. 